\title{
Retrieval of the piecewise-constant mass density in the Bergman wave equation
}

\author{
armand wirgin ${ }^{1}$
}

${ }^{1}$ Laboratoire de Mécanique et d'Acoustique

December 17, 2021

\begin{abstract}
This investigation is concerned with the $2 \mathrm{D}$ acoustic scattering problem of a plane wave propagating in a non-lossy, isotropic, homogeneous fluid host and soliciting a linear, isotropic, macroscopically-homogeneous, generally-lossy, flat-plane layer in which the mass density and wavespeed are different from those of the host. The focus is on the inverse problem of the retrieval of the layer mass density. The data is the transmitted pressure field, obtained by simulation (resolution of the forward problem) in exact, explicit form via the domain integral form of the Bergman wave equation. This solution is exact and more explicit in terms of the mass-density contrast (between the host and layer) than the classical solution obtained by separation of variables. A perturbation technique enables the solution (in its form obtained by the domain integral method) to be cast as a series of powers of the mass density contrast, the first three terms of which are employed as the trial models in the treatment of the inverse problem. The aptitude of these models to retrieve the mass density contrast is demonstrated both theoretically and numerically.
\end{abstract}

\section{Hosted file}

rholayer_short-7.pdf available at https://authorea.com/users/451624/articles/549851retrieval-of-the-piecewise-constant-mass-density-in-the-bergman-wave-equation 